\title{
Combined Therapy of Orthopedic Surgery after Deep Brain Stimulation in Cerebral Palsy Mixed Type - A Case Report -
}

\author{
Hong Souk Park, M.D., Eun Sook Park, M.D., Ph.D., Jin Woo Chang, M.D., Ph.D. ', \\ Ki Seok Lee, M.D., Ph.D. ${ }^{2}$, Young Joo Suh, B.S., Sung-Rae Cho, M.D., Ph.D.
}

Department \& Research Institute of Rehabilitation Medicine, Yonsei University College of Medicine, Departments of ${ }^{1}$ Neurosurgery, ${ }^{2}$ Orthopedic Surgery, Yonsei University College of Medicine, Seoul 120-752, Korea

Dystonia is a symptom defined by involuntary and irregular contractions of the muscles, which cause movement disorders and postural problems. Deep brain stimulation (DBS) in globus pallidus interna (GPi) is a good option for controlling dystonia. DBS has already been shown to have significant effects on primary dystonia as well as Parkinson's disease. Dystonia is very difficult to manage, as seen in cerebral palsy (CP) mixed with spasticity. As CP patients grow, their musculoskeletal problems may require orthopedic surgery. However, the outcome of orthopedic surgery is not usually suitable due to dystonia. Therefore, we attempted to control dystonia through DBS initially and perform orthopedic surgery to correct musculoskeletal deformities after treatment of dystonia. Herein, we report a case that showed remarkable improvement in terms of the dystonia rating scale and gait pattern after combined therapy of DBS and orthopedic surgery.

Key Words Dystonia, Deep brain stimulation, Cerebral palsy

\section{INTRODUCTION}

Dystonia, a symptom involving involuntary and irregular contraction of muscles, causes movement disorders and postural problems and is often found in

Received September 22, 2010; Accepted January 20, 2011

Corresponding author: Sung-Rae Cho

Department of Rehabilitation Medicine, Yonsei University College of Medicine, 134, Shinchon-dong, Seodamun-gu, Seoul 120-752, Korea Tel: +82-2-2228-3715, Fax: +82-2-363-2795, E-mail: srcho918@yuhs.ac

(c) This is an open-access article distributed under the terms of the Creative Commons Attribution Non-Commercial License (http:// creativecommons.org/licenses/by-nc/3.0) which permits unrestricted noncommercial use, distribution, and reproduction in any medium, provided the original work is properly cited.

Copyright ( 2011 by Korean Academy of Rehabilitation Medicine cerebral palsy (CP) mixed with spasticity. ${ }^{1}$ Dystonia in $\mathrm{CP}$ is mainly caused by damage to the extrapyramidal system such as thalamus and basal ganglia, which can occur by hypoxic brain damage or kernicterus. ${ }^{2}$ It is also common for these patients to have later changes in the musculoskeletal system due to spasticity, dyskinesia, and dystonia. However, there remains no permanently effective treatment for patients with severe dystonia, despite their low ability for independent ambulation or activity of daily living.

Therefore, we initially attempted to control dystonia by deep brain stimulation (DBS) and subsequently performed orthopedic surgery to correct musculoskeletal deformities. Herein, we report a CP case that showed re- 
A
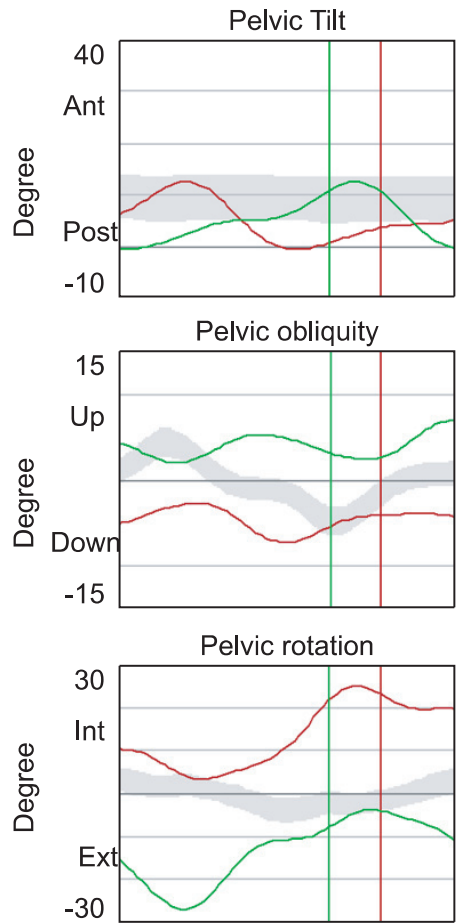

B
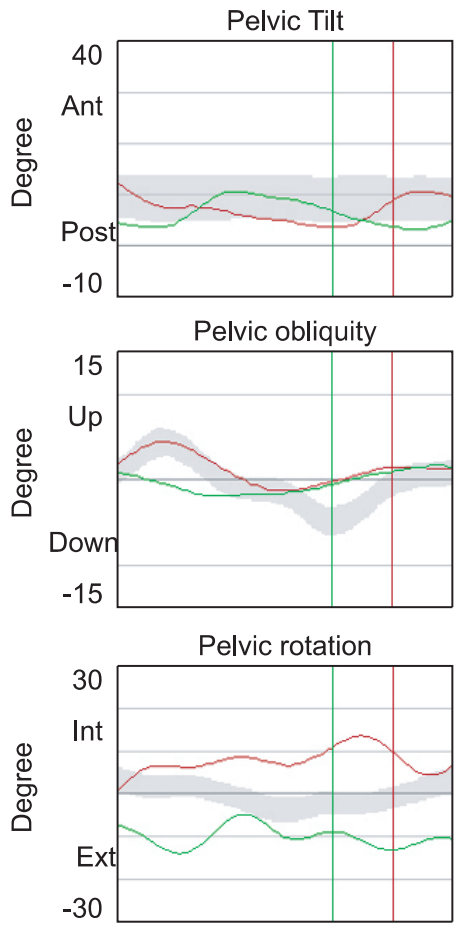

Gait cycle

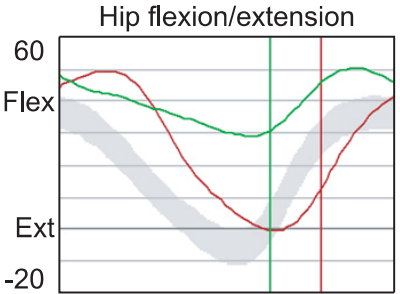

Hip Ab/adduction

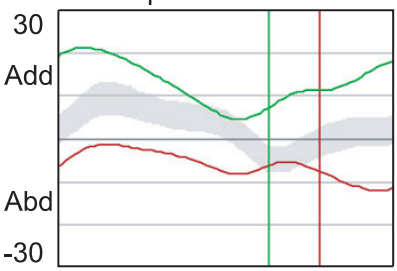

$-30$
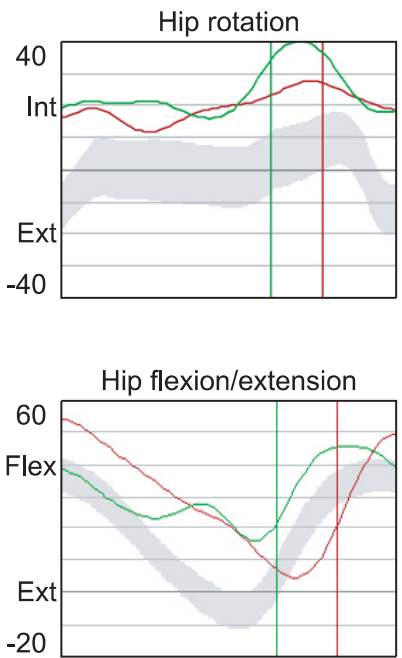

Hip Ab/adduction

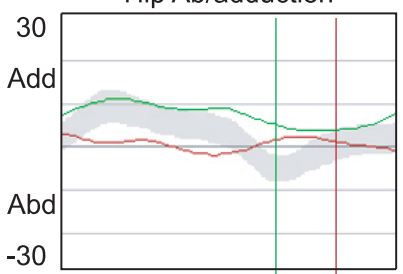

Hip rotation

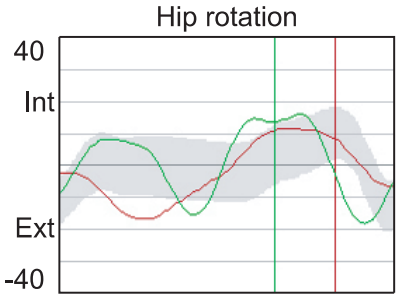

Gait cycle

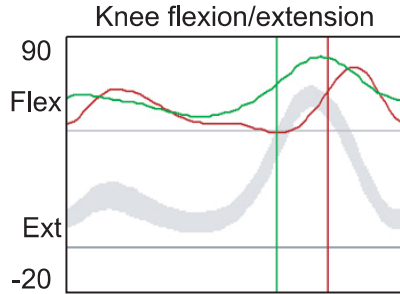

Knee varus
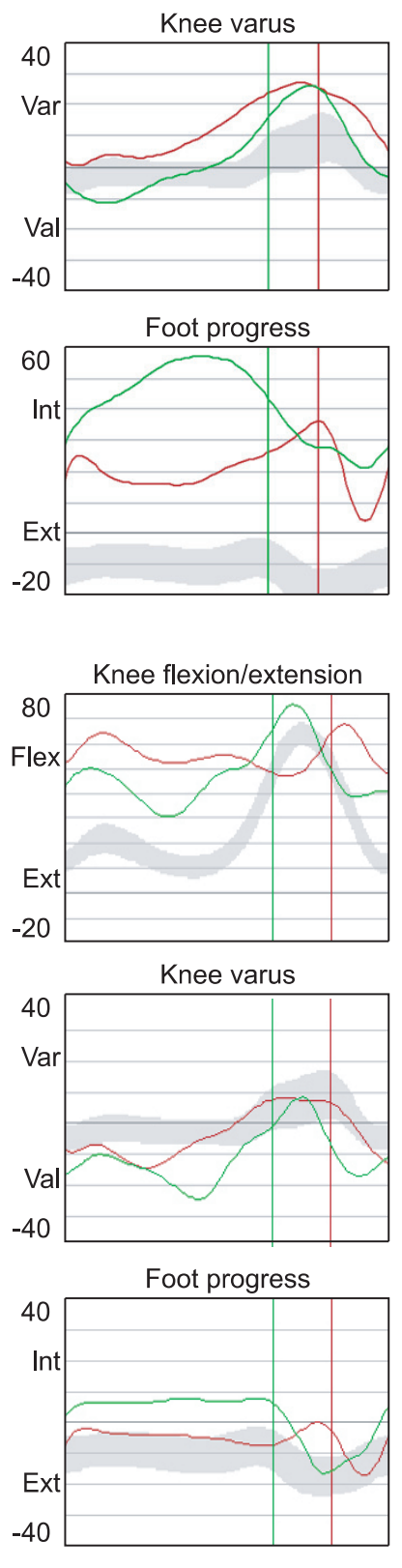

Gait cycle
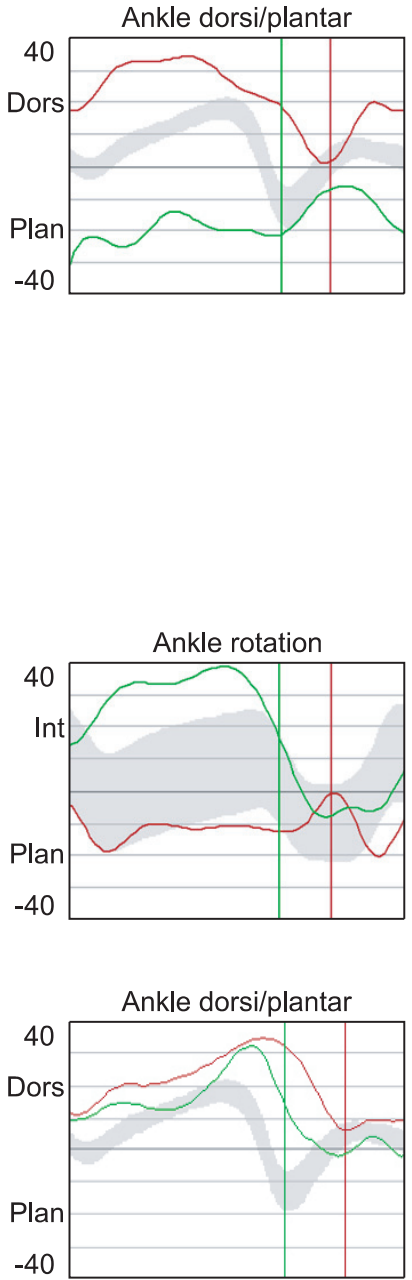

$-40$

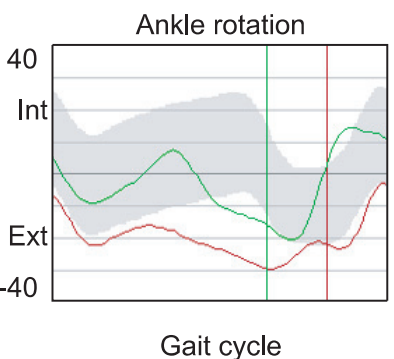

Fig. 1. Kinematic Changes in Gait Analysis after Combined Therapy of DBS and Orthopedic Surgery. When compared with preoperative state (A), pelvic bump sign, asymmetry of pelvic obliquity and ankle plantar flexion deformity were attenuated after orthopedic surgery performed 6 months after treatment of dystonia with DBS (B). DBS: Deep brain stimulation. 
markable improvement in both the dystonia rating scale and gait patterns after combined therapy of DBS and orthopedic surgery.

\section{CASE REPORT}

A 22-year-old male patient came to the orthopedic and rehabilitation clinics at our hospital with right equinovarus and gait disturbance. He had a history of hypoxic brain damage caused by the umbilical cord wrapping around fetal neck at birth, and was diagnosed with $\mathrm{CP}$ at the age of 1 year. He was experiencing severe dystonia in his neck and bilateral upper extremities; the Burke-FahnMarsden dystonia rating scale (DRS) showed 55 points on the dystonia movement scale, and 10 points on the disability scale.

On manual muscle test, the left extremities were within the normal limit, but the right upper and lower extremities only showed grade 3 (Fair). He showed right hip joint flexion contracture and right ankle plantar flexion contracture on the range of motion test. In terms of spasticity, his right upper and lower extremities scored grade 2 on the modified Ashworth scale (MAS). He had 78 points on the modified Barthel index (MBI), and 105 points on the functional independence measure (FIM). He showed asymmetry in the lower extremities and equinovarus in the right ankle on a plain X-ray, and 60 degrees right femoral anteversion, 15.3 degrees left femoral anteversion, 38.9 degrees right tibial torsion and 37.1 degrees left tibial torsion on CT.

In gait analysis, he showed no dissociated movement between the pelvis and lower limbs, so we were able to see the bump sign, and the right pelvis were positioned superiorly to the left pelvis in the coronal plane during the whole gait cycle. Internal rotation of the right pelvis and external rotation of the left pelvis were observed in the transverse plane, and both hip joints showed internal rotation in the sagittal plane. Due to tightness in the hamstring muscle, both knee flexion posture and right ankle planter flexion posture were displayed during the whole gait cycle (Fig. 1-A).

To differentiate from hereditary torsion dystonia, when fragment analysis was performed on the DYT1 gene, the GAG deletion of exon 5 was not observed. Likewise, there were no symptoms, and no findings were observed in blood tests and urinalyses after suspicion of Wilson's disease. In addition, no notable findings were observed on the brain MRI scan, but it was found that uptake of fluodeoxyglucose (FDG) decreased in the bilateral parietal lobe, thalamus and cerebellum on a positron emission tomography (PET) of the brain.

A plan was made for this patient to use DBS in consultation with the department of neurosurgery. After electrode insertion was performed on the globus pallidus interna (GPi), muscle tone decreased. Approximately six months later, right adductor tenotomy and bilateral medial hamstring release were performed in addition to rectus femoris transfer on the right sartorius, tibialis posterior transfer on the right peroneous brevis and derotational osteotomy on the right femur in the department of orthopedic surgery.

The patient was then transferred to the department of rehabilitation medicine and treated with comprehensive rehabilitation, including physical therapy and occupational therapy. The therapeutic outcomes were evaluated through DRS and gait analysis. On the dystonia movement scale, the patient scored 55 before DBS, but 3 months later, he scored 26.5, which was an improvement of $51.9 \%$. One year later, the patient scored 24 , which was an improvement of $56.4 \%$. On the disability scale, the patient scored 10 before surgery and 9 one year after surgery (Table 1). On the gait analysis performed three months after surgery, the anterior tilt of the pelvis decreased in the sagittal plane. In the coronal plane,

Table 1. Changes in Dystonia Rating Scale after Combined Therapy of DBS and Orthopedic Surgery

\begin{tabular}{lccccc}
\hline \multicolumn{1}{c}{ Scale } & Pre-DBS & 3 months & $\begin{array}{c}\text { 6 months } \\
\text { orthopedic } \\
\text { surgery }\end{array}$ & 9 months & 12 months \\
\hline Dystonia movement & 55 & 26.5 & 24.5 & 24 & 24 \\
Disability & 10 & 9 & 9 & 10 & 9 \\
\hline
\end{tabular}

DBS: Deep brain stimulation 
pelvic obliquity almost disappeared. In the transverse plane, pelvic asymmetry and femoral internal rotation were also attenuated. In addition, right ankle plantar flexion was remarkably ameliorated in the sagittal plane (Fig. 1-B).

\section{DISCUSSION}

Dystonia is classified as primary dystonia, which is caused by hereditary or unknown factors, and as secondary dystonia caused by brain damage or Wilson's disease. The DBS of GPi is widely known to be an effective treatment for primary dystonia. ${ }^{3-5}$ However, DBS seems to be not as effective on secondary dystonia as on primary dystonia. ${ }^{3,6}$ On the contrary, Vidailhet et al. ${ }^{7}$ reported that DRS reduced from 44.2 to 34.7 (improvement of $21.5 \%$ ) in 13 patients with dyskinetic CP who underwent bilateral pallidal DBS. In several studies, it was reported that patients with dyskinesia showed an improvement of $30-37 \%$ after undergoing DBS, ${ }^{8,9}$ whereas other studies reported improvement of $0-14 \%{ }^{6,10}$ There may be two important reasons why these studies showed different results. First, in the case of CP mixed type, it is difficult to predict therapeutic effects because dystonia, choreoathetosis, and rigidity occur concurrently. Second, in cases of patients with $\mathrm{CP}$ who have had functional or structural encephalopathy from the developmental process, DBS of GPi tends to stimulate surrounding areas, including the globus pallidis externa (GPe). This may cause dystonia to further worsen ${ }^{7}$. Nevertheless, there have been no reports on the prognostic factors of DBS for CP. Therefore, there is a need to continuously follow up these symptoms in the field of rehabilitation medicine.

This patient showed significant improvement of dystonia after DBS and maintained his muscle tone for a year and longer, which showed that DBS may be an alternative for treating severe dystonia in CP. It remains to be proven whether DBS is effective on dyskinetic CP. So far, there have been no reports on the results of gait analysis and DRS performed on patients with CP mixed with dystonia, spasticity, and musculoskeletal deformity who underwent DBS and orthopedic surgery. This patient showed an improvement in muscle tone after undergoing DBS, but this did not significantly change his gait patterns. After orthopedic surgery, however, his gait patterns largely improved. This implies that DBS for dystonia may be complementary to orthopedic surgery for spasticity, musculoskeletal deformity and gait disturbance in treating CP.

Our report demonstrates that severe dystonia, spasticity, musculoskeletal deformity, and gait disturbance significantly improved in a CP case after combined therapy of DBS and orthopedic surgery.

\section{ACKNOWLEDGEMENTS}

This study was supported by grants from National Research Foundation (NRF-2010-0020408; 2010-0024334) and Stem Cell Research Center of the $21^{\text {st }}$ Century Frontier Research Program (SC-4160) funded by the Ministry of Science and Technology, Republic of Korea.

\section{REFERENCES}

1. Koman LA, Smith BP, Shilt JS. Cerebral palsy. Lancet 2004; 363: 1619-1631

2. Monbaliu E, Ortibus E, Roelens F, Desloovere K, Deklerck J, Prinzie P, de Cock P, Feys H. Rating scales for dystonia in cerebral palsy: reliability and validity. Dev Med Child Neurol 2010; 52: 570-575

3. Cif L, El Fertit H, Vayssiere N, Hemm S, Hardouin E, Gannau A, Tuffery S, Coubes P. Treatment of dystonic syndromes by chronic electrical stimulation of the internal globus pallidus. J Neurosurg Sci 2003; 47: 5255

4. Vidailhet M, Vercueil L, Houeto JL, Krystkowiak P, Benabid AL, Cornu P, Lagrange C, Tezenas du Montcel S, Dormont D, Grand S, et al. Bilateral deepbrain stimulation of the globus pallidus in primary generalized dystonia. N Engl J Med 2005; 352: 459-467

5. Kupsch A, Benecke R, Muller J, Trottenberg T, Schneider GH, Poewe W, Eisner W, Wolters A, Muller JU, Deuschl G, et al. Pallidal deep-brain stimulation in primary generalized or segmental dystonia. $\mathrm{N}$ Engl J Med 2006; 355: 1978-1990

6. Vercueil L, Krack P, Pollak P. Results of deep brain stimulation for dystonia: a critical reappraisal. Mov Disord 2002; 17 Suppl 3: S89-93

7. Vidailhet M, Yelnik J, Lagrange C, Fraix V, Grabli D, Thobois S, Burbaud P, Welter ML, Xie-Brustolin J, Braga MC, et al. Bilateral pallidal deep brain stimulation for the treatment of patients with dystonia- 
choreoathetosis cerebral palsy: a prospective pilot study. Lancet Neurol 2009; 8: 709-717

8. Zorzi G, Marras C, Nardocci N, Franzini A, Chiapparini L, Maccagnano E, Angelini L, Caldiroli D, Broggi G. Stimulation of the globus pallidus internus for childhood-onset dystonia. Mov Disord 2005; 20: 1194-1200

9. Starr PA, Turner RS, Rau G, Lindsey N, Heath S, Volz M, Ostrem JL, Marks WJ Jr. Microelectrode-guided implantation of deep brain stimulators into the globus pallidus internus for dystonia: techniques, electrode locations, and outcomes. J Neurosurg 2006; 104: 488501

10. Krauss JK, Loher TJ, Weigel R, Capelle HH, Weber S, Burgunder JM. Chronic stimulation of the globus pallidus internus for treatment of non-DYT1 generalized dystonia and choreoathetosis: 2-year follow up. J Neurosurg 2003; 98: 785-792 